\title{
Molecular phylogeny and RNA secondary structure of Fusarium species with different lifestyles
}

\author{
Barik $\mathbf{B P}^{1}$, Tayung $\mathbf{K}^{2 *}$ and Jagadev $\mathbf{P N}^{3}$ \\ ${ }^{1}$ Department of Bioinformatics and DBT-Bioinformatics Infrastructure Facility, North Orissa University, Takatpur, \\ Baripada (India) \\ ${ }^{2}$ Department of Botany and Bioinformatics, North Orissa University, Takatpur, Baripada (India) \\ ${ }^{3}$ Department of Plant Breeding and Genetics, Orissa University of Agriculture and Technology, Bhubaneswar (India)
}

Barik BP, Tayung K, Jagadev PN 2011 - Molecular phylogeny and RNA secondary structure of Fusarium species with different lifestyles. Plant Pathology \& Quarantine 1(2), 205-219, doi $10.5943 / \mathrm{ppq} / 1 / 2 / 5$

The definition of endophytes ranges from symbiotic to balanced antagonism and/or latent plant pathogens. This indicates a close affiliation between endophytes and pathogens but molecular evidence confirming these relationships are still to be elucidated. We investigated the relationship of endophytic, saprobic and pathogenic Fusarium species as a model genus based on ITS sequences and ITS2 secondary structure analysis. Altogether 212 Fusarium species mostly named in GenBank with ranging lifestyles were used in this study. Species deposited in GenBank were found to be often wrongly named based on sequence comparison as species with the same names were polyphyletic, while differently named species often had the same sequences. Phylogenetic analysis by three different methods (UPGMA, NJ and MP) revealed that similar named species clustered together, but did not form well distinct clades reflective of their lifestyles. Several species were polyphyletic, while several other unrelated species appear to share the same ITS sequences. Since ITS rDNA sequence based phylogeny did not showed distinct relationships, ITS 2 consensus secondary structures were used. Endophytes showed close molecular structural affinity with pathogens but structural features of saprobes was very different. Although care must be taken when using sequences from named Fusarium species in GenBank this study provides molecular evidence that endophytes share a close relationship with pathogens and agrees with the assumption that endophytes are latent pathogens.

Key words - Fusarium spp. - Lifestyle - Phylogeny - Secondary structures analysis

\section{Article details}

Received 15 September 2011

Accepted 11 October 2011

Published online 19 November 2011

*Corresponding author: Tayung $\mathrm{K}$ - e-mail - kumanandbotnou@ @ rediffmail.com

\section{Introduction}

There have been numerous reports on endophytic fungi colonizing various plant hosts and speculation on their functional roles in the environment (Hyde \& Soytong 2008, Albrectsen et al 2010, Saikkonen et al 2010). Most definitions regard endophytes as internal plant tissue colonizers that do not cause any disease symptoms. Such a definition implies a symbio- tic or mutualistic relationship between the host plant and the endophytic microbe. There have however, been differences in opinion concerning the relationships that exist between the endophyte and their respective host. Strobel (2002) suggested that the relationship can range from borderline pathogenic, to commensalism and to a symbiotic relationship. There are also studies that have explored relationships 
between endophytes and their roles as saprobes (Hyde et al. 2007, Promputtha et al. 2007, 2010, Purahong \& Hyde 2011). There are also many examples showing endophytes to be latent pathogens (Hyde \& Soytong 2008). In fact, several fungal taxa reported as endophytes closely resemble plant pathogenic fungi. Such findings suggest an inherent genomic modulation and adaptability in endophytic fungi. Even though there are hypotheses providing evidence for these transitional forms; molecular level investigations with regard to their different lifestyles are few (Promputtha et al. 2007). We have attempted to study the relationships between endophytic, saprobic and pathogenic Fusarium species as a model organism based on nuclear Internal Transcribed Spacer (ITS) sequences and RNA secondary structures study.

Fusarium is a highly diverse genus with species exhibiting many different lifestyles (Summerell et al 2010, 2011. They are generally regarded as plant pathogens causing serious diseases (Amata et al 2010). Some are also reported to cause diseases in humans especially in immuno-compromised patients, while others live as saprobes in the soil and play a role in nutrient recycling. Fusarium species have commonly been isolated as endophytes and reported from various plant hosts (Strobel and Daisy 2003, Xu et al. 2008, Deng et al. 2009). The existence of Fusarium species occupying different lifestyles is confusing because morphologically they cannot be welldifferentiated. Molecular techniques have often been used to identify Fusarium species (Amata 2010, Summerell et al 2010, 2011), but there is little information on their phylogeny and variations at molecular level considering isolates existing in different forms.

The Internal Transcribed Spacer (ITS) regions of rDNA have commonly been used in species differentiation. They form specific secondary structures which are needed for correct recognition of cleavage sites and provide the binding sites for nucleolar proteins and RNAs during ribosome maturation (Hausner and Xang 2005). ITS regions that comprised two transcribed intergenic spacers (ITS1 and ITS2) have not only been used for phylogenetic and taxonomic studies of fungi but also for species delimitation and ecological analysis (Pinto et al. 2004; Anderson and Perkin 2007). The ITS2 region has been found to vary in both primary sequences and secondary structures, so it could be used as possible marker in molecular systematics and phylogenetic reconstruction (Schultz \& Wolf 2009). Several researchers have already demonstrated the potential applications of ITS2 for taxonomic classification and phylogenetic reconstruction at both the genus and species levels for eukaryotes, including animals, plants and fungi (Coleman 2007, Miao et al. 2008, Keller et al. 2009). Secondary structural data analysis of this region can also improve the phylogenetic resolution to a considerable extent (Keller et al. 2008). Since structures are more conserved than sequences, the aim of this study was to infer phylogenetic relationships and to address variations among Fusarium spp. having different lifestyles based on ITS sequence and structure analysis.

\section{Materials and Methods}

\section{Isolation of the source organisms}

Fusarium strains occurring in different habitats were isolated from various sources (Table 1). Endophytic strains were obtained from their respective hosts by surface sterilization procedure. The technique used for isolation was as follows: Barks samples were immersed sequentially in $70 \%$ ethanol for $3 \mathrm{~min}$ and $0.5 \% \mathrm{NaOCl}$ for $1 \mathrm{~min}$ and rinsed thoroughly with sterile distilled water. The excess water was dried under laminar airflow chamber. Then, with sterile scalpel, outer tissues were removed and the inner tissues were carefully dissected and placed on Petri-dishes containing Potato Dextrose Agar (PDA) medium and incubated at $25^{\circ} \mathrm{C}$. Saprobic strains were obtained by dilution plate method. Soil samples were serially diluted and each dilution was plated on Potato Dextrose Agar medium and incubated at $30^{\circ} \mathrm{C}$ for week. Pathogenic strains comprised of one plant pathogen and one human pathogen. The plant pathogenic strain was obtained from the experimental farm of Orissa University of Agriculture and Technology, Odisha, India and were isolated from an infected immature coconut. The human pathogenic strain was obtained from the Microbiology Division of Kasturaba Medical 
Table 1 Fusarium spp. isolated from various sources used in present study.

\begin{tabular}{|c|c|c|c|}
\hline Fusarium spp. & Isolation source & Lifestyle & $\begin{array}{l}\text { GenBank } \\
\text { Accession No. }\end{array}$ \\
\hline F. solani & Taxus baccata bark & Endophyte & FJ719812.1 \\
\hline F. proliferatum & Ipomoea carnea bark & Endophyte & HM145946.1 \\
\hline F. proliferatum & Immature coconut & Pathogen & GU363955.1 \\
\hline F. solani & Corneal scrape & Pathogen & HM145945.1 \\
\hline F. oxysporum & Grassland soil & Saprobe & GU363956.1 \\
\hline F. incarnatum & River belt soil & Saprobe & HQ221741.1 \\
\hline
\end{tabular}

College, Manipal University, Manipal, India. The strain was isolated on Sabouraud Dextrose Agar (SDA) medium from corneal scrape of 38 years old male patient.

\section{Isolation of genomic DNA, PCR amplifica- tion and sequencing}

Total genomic DNA was extracted from mycelia of the fungi using a CTAB method (Cai et al. 2005). DNA amplification was performed by PCR. The PCR was set up using the following components: $5 \mu \mathrm{L}$ Buffer $(10 \times), 3 \mu \mathrm{L}$ $\mathrm{MgCl} 2(25 \mathrm{mM}), 1 \mu \mathrm{L}$ dNTPs $(10 \mathrm{mM}), 1.5 \mu \mathrm{L}$ Taq Polymerase $(5 \mathrm{U}), 1.5 \mu \mathrm{L}$ Forward primer $(10 \mu \mathrm{M}), 1.5 \mu \mathrm{L}$ Reverse Primer $(10 \mu \mathrm{M}), 3 \mu \mathrm{L}$ DNA template and $34.7 \mu \mathrm{L}$ distilled water. Initial denaturation was at $95^{\circ} \mathrm{C}$ for 5 min denaturation, annealing and elongation were done at $95^{\circ} \mathrm{C}$ for $1 \mathrm{~min}, 52^{\circ} \mathrm{C}$ for $30 \mathrm{sec}$ and $72^{\circ} \mathrm{C}$ for 1 min, respectively in 45 cycles. Final extension was at $72^{\circ} \mathrm{C}$ for $10 \mathrm{~min}$ and hold at $4{ }^{\circ} \mathrm{C}$. For amplification of ITS-rDNA region ITS4 and ITS5 primers were used according to the method described by White et al. (1990). The PCR product, spanning approximately 500-600bp was checked on $1 \%$ agarose electrophoresis gel. It was then purified using a quick spin column and buffers (washing buffer and elution buffer) according to the manufacturer's protocol (QIA quick gel extraction kit Cat No. 28706). DNA sequencing was performed using the above mentioned primers in an Applied Biosystem 3130xl analyzer.

\section{Taxon sampling}

Six Fusarium strains were obtained as isolates. The sequences were annotated and submitted to GenBank. ITS rDNA sequence data from 2437 Fusarium species were also retrieved from GenBank (on 26-12-2010). The sequences were also trimmed for ITS2 using annotation tools based on Hidden Markov Mo- del (Keller et al. 2009). The sequences were filter searched and 206 sequences were selected based on their mode of occurrence as endophytes, saprobes and pathogens. Altogether 212 ITS rDNA sequences were considered for phylogenetic analysis and same numbers of ITS2 sequences were used for secondary structure study.

\section{Sequence alignment and phylogenetic ana- lyses}

Multiple sequence alignments were performed using CLUSTALW software utilizing default settings. ITS rDNA sequences were used for phylogenetic relationship inferences. Phylogenetic trees were generated by three different methods (UPGMA, NJ and MP) by MEGA 4.0 (Tamura et al. 2007). All characters were equally weighted and unordered. Alignment gaps were treated as missing data. The optimal trees with the sum of branch length were 0.47152190 for UPGMA and 0.48761537 for NJ. The percentage of replicate trees in which the associated taxa clustered together in the bootstrap was 500 replicates. The evolutionary distances were computed using the Maximum Composite Likelihood method and are in the units of the number of base substitutions per site. All positions containing gaps and missing data were eliminated from the dataset (complete deletion option). In the MP method, the tree was generated from 344 most parsimonious trees. The consistency index was 0.5828 22 , the retention index was 0.979115 and the composite index was 0.570650 . The MP tree was obtained using the Close-Neighbor-Interchange algorithm. There were a total of 238 positions in the final dataset, out of which 65 were parsimony informative.

\section{Consensus RNA secondary structures pre- diction}


The ITS2 sequences of Fusarium occurring as endophytes, saprobes and pathogens (plant pathogenic and human pathogenic) were used to generate consensus RNA secondary structures using LocARNA version 1.5.2 (Smith et al. 2010). It performed multiple alignments and generated consensus secondary structures of each category using realistic energy model for RNAs. The structures were observed and variations were analyzed amongst different groups.

\section{Results and Discussion}

\section{Isolation, identification and retrieval of $\mathrm{Fu}$ - sarium spp.}

Six Fusarium strains were isolated from various sources; two strains were endophytic, two were saprobic and one each was a phytopathogen and human pathogen (Table 1). All strains were morphologically and microscopically identified as Fusarium species. Species identification of Fusarium using morphological traits has always been a difficult task for mycologists because characteristics like mycelial pigmentation, formation, shape and size of conidia are unstable and highly dependent on composition of media and environmental conditions. Phenotypic variation is abundant and considerable expertise is required to distinguish between closely related species (Nelson 1983). Therefore molecular characterization was carried out based on ITS rDNA data. The strains were sequenced and species level confirmations were done by BLAST search analysis. Among several molecular techniques, ITS rDNA sequence analysis has been a method of choice for species confirmation because they are conserved regions of the genome and genetically variable and have proven to be very useful for investigating relationships between fungi at all taxonomic levels including genus and species (Bruns et al. 1991). All the sequences were deposited in GenBank and GenBank index numbers were obtained. In addition, 206 species of Fusarium with ITS2 sequences were retrieved from ITS2 database based on their habitat and locations (Table 2).

\section{Phylogenetic analyses of Fusarium spp.}

A total of 212 ITS rDNA sequences of Fusarium from different lifestyles were ob- tained as final dataset for phylogenetic study. Sequence analysis of the ITS region of nuclear ribosomal DNA has been widely used for taxonomic placement (Guo et al. 2001, Wang et al. 2005) and phylogenetic diversity of fungi (Tejesvi et al. 2009). This region has also been used in taxonomy and phylogeny of Fusarium species (Schroers et al. 2009). Phylogenetic trees were generated by three different methods (UPGMA, NJ and MP) using MEGA 4.0.2 (Tamura et al. 2007). All the trees showed that more or less similar species of Fusarium clustered together but did not form distinct clades as per their lifestyles. One of the most parsimonious trees showing evolutionary relationships among Fusarium spp. is shown in Figure 1. The result also indicated that several species appear to be polyphyletic and several other unrelated species appear to share the same ITS sequences. This may be due to wrongly named species being submitted to GenBank as in other genera (Cai et al. 2009). Endophytic, saprobic and pathogenic taxa did not cluster into separate groups even in the case of similarly named species. This was particularly obvious in common species of Fusarium such as F. oxysporum and $F$. solani. Several workers have however clearly differentiated endophytic and pathogenic fungi by analyzing the ITS regions (Photita et al. 2005, Wei et al. 2007b). Such findings do not agree with our result, where consideration of ITS rDNA sequence phylogeny did not elucidate variations among closely related species of Fusarium based on their lifestyles. The ambiguity may be due to consideration of many sequences of Fusarium species which is genetically diverse with different lifestyles or the fact that they are wrongly named in GenBank. There are also significant drawbacks in using the ITS region such as (i) insufficient hypervariability to distinguish the various species in the Fusarium species complexes; (ii) its failure to distinguish between closely related species (sibling species) and (iii) problems with the reliability of the ITS sequences deposited in the reference databases (e.g., GenBank/EMBL/DDBJ) (Nilsson et al. 2006, Cai et al 2009). Moreover, primary sequences of ITS regions often contain insertions and deletions (indels) making alignment difficult beyond infraspecific levels (Kruger \& Gargas, 2008). Since consideration 
Table 2 Fusarium spp. used in the present study with their isolation sources, lifestyles and GenBank numbers.

\begin{tabular}{|c|c|c|c|}
\hline Species & Source & Lifestyle & GenBank Index \\
\hline F. solani* & Taxus baccata & Endophyte & 224551656 \\
\hline F. proliferatum* & Ipomea carnea & Endophyte & 296410105 \\
\hline F. solani & Campotheca accuminta & Endophyte & 117650700 \\
\hline F. solani & Nothapodytes nimmoniana & Endophyte & 205319823 \\
\hline F. solani & Rhizophoraceae sp. & Endophyte & 291293624 \\
\hline F. solani & Agarwood & Endophyte & 296042316 \\
\hline F. solani & Eleuterococcus seaticosus & Endophyte & 299790610 \\
\hline F. solani & Triplaris cumingiana & Endophyte & 304333705 \\
\hline F. solani & Mulberry Roots & Endophyte & 186694316 \\
\hline F. equiseti & Curcuma longa & Endophyte & 144905075 \\
\hline F. equiseti & Vitis vinifera & Endophyte & 154721227 \\
\hline F. equiseti & Mulberry Roots & Endophyte & 186694315 \\
\hline F. equiseti & - & Endophyte & 222477058 \\
\hline F. equiseti & Holcus lanatus & Endophyte & 240936106 \\
\hline F. equiseti & Agarwood & Endophyte & 296042314 \\
\hline F. equiseti & Agarwood & Endophyte & 296042333 \\
\hline F. equiseti & Piper reticulatum & Endophyte & 304333726 \\
\hline F. oxysporum & Paris polyphylla & Endophyte & 146217051 \\
\hline$F$. oxysporum & Mangrove & Endophyte & 159024208 \\
\hline F. oxysporum & Dioscorea zingiberensis & Endophyte & 170785449 \\
\hline F. oxysporum & Mulberry Roots & Endophyte & 186694314 \\
\hline F. oxysporum & Mexican Yew Leaf & Endophyte & 203285112 \\
\hline F. oxysporum & Nothapodytes nimmoniana & Endophyte & 205319842 \\
\hline F. oxysporum & Palicourea tetraphylla & Endophyte & 217331371 \\
\hline F. redolens & Paris polyphylla & Endophyte & 146217055 \\
\hline$F$. redolens & Rehmannia glutinosa & Endophyte & 299851415 \\
\hline F. redolens & Rehmannia glutinosa & Endophyte & 299851446 \\
\hline F. tricinctum & Centaurea stoebe & Endophyte & 148357869 \\
\hline F. tricinctum & Dendrobium & Endophyte & 295855229 \\
\hline F. tricinctum & Dendrobium & Endophyte & 295855230 \\
\hline F. tricinctum & Dendrobium & Endophyte & 295855231 \\
\hline F. tricinctum & Dendrobium & Endophyte & 295855242 \\
\hline F. pulverosum & Centaurea stoebe & Endophyte & 152060774 \\
\hline F. poae & Centaurea stoebe & Endophyte & 152060775 \\
\hline F. sporotrichioides & Centaurea stoebe & Endophyte & 152060776 \\
\hline F. incarnatum & Vitis vinifera & Endophyte & 154721229 \\
\hline F. culmorum & Coffee Plant & Endophyte & 157986165 \\
\hline F. culmorum & Holcus lanatus & Endophyte & 240936141 \\
\hline$F$. lateritium & Mangrove & Endophyte & 199599783 \\
\hline$F$. lateritium & Mexican Yew Leaf & Endophyte & 203285125 \\
\hline F. lateritium & Mexican Yew Leaf & Endophyte & 203285140 \\
\hline F. chlamydosporum & Mexican Yew Leaf & Endophyte & 203285050 \\
\hline F. chlamydosporum & Mexican Yew Leaf & Endophyte & 203285051 \\
\hline F. chlamydosporum & Mexican Yew Leaf & Endophyte & 203285052 \\
\hline F. chlamydosporum & Mexican Yew Leaf & Endophyte & 203285068 \\
\hline F. chlamydosporum & Dracaena sp. & Endophyte & 295814524 \\
\hline F. oxysporum & Nothapodytes nimmoniana & Endophyte & 205319826 \\
\hline F. aquaeductuum & Mangrove & Endophyte & 223016912 \\
\hline F. polyphialidicum & Hevea brasilensis & Endophyte & 229560273 \\
\hline F. polyphialidicum & Pharus virescens & Endophyte & 304333709 \\
\hline F. polyphialidicum & Ananas comosus & Endophyte & 304333925 \\
\hline F. larvarum & Pinus halpensis & Endophyte & 298356922 \\
\hline F. arthrosporioides & Taxus cuspidata & Endophyte & 46318076 \\
\hline F. oxysporum* & Soil & Saprobe & 283854593 \\
\hline F. incarnatum* & Soil & Saprobe & 312064792 \\
\hline F. oxysporum & Soil & Saprobe & 11692812 \\
\hline F. oxysporum & Soil & Saprobe & 148733622 \\
\hline
\end{tabular}


Table 2 (continued) Fusarium spp. used in the present study with their isolation sources, lifestyles and GenBank numbers.

\begin{tabular}{|c|c|c|c|}
\hline Species & Source & Lifestyle & GenBank Index \\
\hline F. oxysporum & $\begin{array}{l}\text { Soil } \\
\end{array}$ & Saprobe & 148733623 \\
\hline F. oxysporum & Soil & Saprobe & 148733625 \\
\hline F. oxysporum & Soil & Saprobe & 162285917 \\
\hline F. oxysporum & Soil & Saprobe & 204463578 \\
\hline F. oxysporum & Soil & Saprobe & 237769607 \\
\hline F. oxysporum & Soil & Saprobe & 261277540 \\
\hline F. oxysporum & Soil & Saprobe & 28208780 \\
\hline F. oxysporum & Soil & Saprobe & 28208780 \\
\hline F. oxysporum & Soil & Saprobe & 289119520 \\
\hline F. oxysporum & Soil & Saprobe & 289718953 \\
\hline F. oxysporum & Soil & Saprobe & 289780330 \\
\hline F. oxysporum & Soil & Saprobe & 304651424 \\
\hline F. oxysporum & Soil & Saprobe & 304651425 \\
\hline F. oxysporum & Soil & Saprobe & 304651428 \\
\hline F. oxysporum & Soil & Saprobe & 304651429 \\
\hline F. oxysporum & Soil & Saprobe & 60593117 \\
\hline F.incarnatum & Soil & Saprobe & 134290455 \\
\hline F.incarnatum & Soil & Saprobe & 262069425 \\
\hline F. equiseti & Soil & Saprobe & 148733624 \\
\hline F. equiseti & Soil & Saprobe & 162285925 \\
\hline F. equiseti & Soil & Saprobe & 219523076 \\
\hline F. equiseti & Soil & Saprobe & 289718950 \\
\hline F. tricinctum & Soil & Saprobe & 148733626 \\
\hline F. tricinctum & Soil & Saprobe & 148733629 \\
\hline F. tricinctum & Soil & Saprobe & 148733631 \\
\hline F. tricinctum & Soil & Saprobe & 148733632 \\
\hline F. tricinctum & Soil & Saprobe & 237769552 \\
\hline F. tricinctum & Soil & Saprobe & 261277537 \\
\hline F. solani & Soil & Saprobe & 149132031 \\
\hline F. solani & Soil & Saprobe & 197717741 \\
\hline F. solani & Soil & Saprobe & 204463676 \\
\hline F. solani & Soil & Saprobe & 215513486 \\
\hline F. solani & Soil & Saprobe & 215513487 \\
\hline F. solani & Soil & Saprobe & 215513488 \\
\hline F. solani & Soil & Saprobe & 215513490 \\
\hline F. solani & Soil & Saprobe & 215513491 \\
\hline F. solani & Soil & Saprobe & 223366136 \\
\hline F. solani & Soil & Saprobe & 237769596 \\
\hline F. solani & Soil & Saprobe & 237769597 \\
\hline F. solani & Soil & Saprobe & 256856198 \\
\hline F. solani & Soil & Saprobe & 289780325 \\
\hline F. solani & Soil & Saprobe & 289780326 \\
\hline F. solani & Soil & Saprobe & 289780327 \\
\hline F. solani & Soil & Saprobe & 289780328 \\
\hline F. solani & Soil & Saprobe & 289780329 \\
\hline F. solani & Soil & Saprobe & 304651421 \\
\hline F. sporotrichioides & Soil & Saprobe & 187235530 \\
\hline F. delphinoides & Soil & Saprobe & 294653635 \\
\hline F. oxysporum & Soil & Saprobe & 297185611 \\
\hline F. brachygibbosum & Soil & Saprobe & 298684054 \\
\hline F. proliferatum* & Immature Coconut & Phytopathogen & 283854592 \\
\hline F. cerealis & Plant with Head Blight & Phytopathogen & 91719251 \\
\hline F. lunulosporum & Plant with Head Blight & Phytopathogen & 91719250 \\
\hline F. brasilicum & Plant with Head Blight & Phytopathogen & 91719243 \\
\hline F. mesoamericanum & Plant with Head Blight & Phytopathogen & 91719226 \\
\hline F. oxysporum & Gladiolus & Phytopathogen & 82622243 \\
\hline
\end{tabular}


Table 2 (continued) Fusarium spp. used in the present study with their isolation sources, lifestyles and GenBank numbers.

\begin{tabular}{|c|c|c|c|}
\hline Species & Source & Lifestyle & GenBank Index \\
\hline F. oxysporum & Confier norsum & Phytopathogen & 68508671 \\
\hline F. oxysporum & Confier norsum & Phytopathogen & 68508670 \\
\hline F. oxysporum & Asparagus root & Phytopathogen & 68267976 \\
\hline F. oxysporum & Lotus & Phytopathogen & 62948004 \\
\hline F. pseudograminearum & Wheat & Phytopathogen & 32135112 \\
\hline F. oxysporum & Bean & Phytopathogen & 302311065 \\
\hline$F$. poae & Wheat & Phytopathogen & 288965987 \\
\hline$F$. poae & Zea mays & Phytopathogen & 83272581 \\
\hline F. lateritium & Hezel nut & Phytopathogen & 293321577 \\
\hline F. lateritium & Wheat & Phytopathogen & 288965977 \\
\hline F. solani & Daucus carota & Phytopathogen & 284507210 \\
\hline F. incarnatum & Banana & Phytopathogen & 83595204 \\
\hline F. oxysporum & Chickpea wilt & Phytopathogen & 260101097 \\
\hline F. polyphialidicum & Bean & Phytopathogen & 224472853 \\
\hline F. tricinctum & Kiwi fruit & Phytopathogen & 209360972 \\
\hline F. oxysporum & Cotton & Phytopathogen & 194580114 \\
\hline F. oxysporum & Watermelon & Phytopathogen & 183673382 \\
\hline F. chlamydosporum & Guava & Phytopathogen & 295814506 \\
\hline F. chlamydosporum & Ginger & Phytopathogen & 239819638 \\
\hline F. oxysporum & Lentil & Phytopathogen & 161876806 \\
\hline F. oxysporum & Citrus sinensis & Phytopathogen & 157073411 \\
\hline$F$. sinensis & Wheat & Phytopathogen & 156254128 \\
\hline F. langsethiae & Wheat & Phytopathogen & 151335753 \\
\hline F. asiaticum & Wheat & Phytopathogen & 148596890 \\
\hline F. asiaticum & Wheat & Phytopathogen & 148596892 \\
\hline F. virguliforme & soybean & Phytopathogen & 148535005 \\
\hline F. virguliforme & Glycine $\max$ & Phytopathogen & 57117619 \\
\hline F. tucumaniae & Soybean & Phytopathogen & 148534981 \\
\hline F. tucumaniae & Soybean & Phytopathogen & 62528633 \\
\hline F. phaseoli & Soybean & Phytopathogen & 148534979 \\
\hline F. phaseoli & Soybean & Phytopathogen & 37788533 \\
\hline F. cuneirostrum & Soybean & Phytopathogen & 148534976 \\
\hline F. cuneirostrum & Soybean & Phytopathogen & 148534977 \\
\hline F. brasiliense & Soybean & Phytopathogen & 148534971 \\
\hline F. redolens & Allium fistulosum & Phytopathogen & 148277513 \\
\hline$F$. redolens & Ginger & Phytopathogen & 239819656 \\
\hline F. sporotrichioides & Solanaceous plant & Phytopathogen & 134103851 \\
\hline F. equiseti & Wheat & Phytopathogen & 126567419 \\
\hline F. equiseti & Bamboo & Phytopathogen & 157704073 \\
\hline F. oxysporum & Solanum tuberosum & Phytopathogen & 116710911 \\
\hline F. oxysporum & Agave tequilana & Phytopathogen & 157922341 \\
\hline F. solani & Sorghum & Phytopathogen & 139538584 \\
\hline F. solani & Lotus japonicus & Phytopathogen & 146219020 \\
\hline F. oxysporum & Banana & Phytopathogen & 114329283 \\
\hline F. culmorum & Wheat & Phytopathogen & 126567411 \\
\hline F. culmorum & Wheat & Phytopathogen & 126567412 \\
\hline F. oxysporum & Confier norsum & Phytopathogen & 68508669 \\
\hline F. solani* & Cornea & Human Pathogen & 296410104 \\
\hline F. culmorum & Cystric fibrousis patient & Human Pathogen & 85375905 \\
\hline F. solani & Human & Human Pathogen & 134269487 \\
\hline F. solani & Human Nail & Human Pathogen & 134269492 \\
\hline F. solani & Human Skin & Human Pathogen & 134269503 \\
\hline F. solani & Cornea & Human Pathogen & 145909935 \\
\hline F. solani & Contact lens & Human Pathogen & 198401846 \\
\hline F. solani & Contact lens & Human Pathogen & 198401859 \\
\hline F. solani & Diabetic foot & Human Pathogen & 45862178 \\
\hline F. solani & Nails & Human Pathogen & 256575429 \\
\hline
\end{tabular}


Table 2 (continued) Fusarium spp. used in the present study with their isolation sources, lifestyles and GenBank numbers.

\begin{tabular}{|c|c|c|c|}
\hline Species & Source & Lifestyle & GenBank Index \\
\hline F. oxysporum & Onychomycosis & Human Pathogen & 194339137 \\
\hline F. oxysporum & Contact lens & Human Pathogen & 198401858 \\
\hline F. oxysporum & Patient with keratitis & Human Pathogen & 256575427 \\
\hline F. oxysporum & Bronchial secretions & Human Pathogen & 256575431 \\
\hline F. oxysporum & Blood infecion & Human Pathogen & 256575437 \\
\hline F. dimerum & Cornea & Human Pathogen & 198401865 \\
\hline F. dimerum & Keratitis & Human Pathogen & 256575433 \\
\hline F. dimerum & Clinical source & Human Pathogen & 148535529 \\
\hline F. incarnatum & Cornea & Human Pathogen & 164654195 \\
\hline F. solani & Cornea & Human Pathogen & 164654201 \\
\hline F. mangiferae & Bronchial secretions & Human Pathogen & 256575432 \\
\hline F. nelsonii & Human & Human Pathogen & 262476204 \\
\hline F. nelsonii & Human & Human Pathogen & 262476206 \\
\hline F. brachygibbosum & Human & Human Pathogen & 262476220 \\
\hline F. armeniacum & Human & Human Pathogen & 262476232 \\
\hline F. flocciferum & Human & Human Pathogen & 262476235 \\
\hline F. equiseti & Human & Human Pathogen & 262476540 \\
\hline F. equiseti & Human & Human Pathogen & 262476542 \\
\hline F. equiseti & Human & Human Pathogen & 262476547 \\
\hline F. equiseti & Human & Human Pathogen & 262476549 \\
\hline F. equiseti & Human & Human Pathogen & 262476553 \\
\hline F. equiseti & Human & Human Pathogen & 262476592 \\
\hline F. equiseti & Human & Human Pathogen & 262476595 \\
\hline F. equiseti & Human & Human Pathogen & 262476601 \\
\hline F. equiseti & Human & Human Pathogen & 262476611 \\
\hline F. lacertarum & Human & Human Pathogen & 262476541 \\
\hline F. concolor & Human & Human Pathogen & 262476622 \\
\hline F. virguliforme & Human & Human Pathogen & 270155615 \\
\hline F. tucumaniae & Human & Human Pathogen & 270155616 \\
\hline F. polyphialidicum & Keratitis & Human Pathogen & 39725403 \\
\hline F. solani & Trachea & Human Pathogen & 56578581 \\
\hline F. solani & Ocular sources & Human Pathogen & 198401863 \\
\hline F. oxysporum & Onychomycosis & Human Pathogen & 194339138 \\
\hline F. oxysporum & Onychomycosis & Human Pathogen & 194339139 \\
\hline F. oxysporum & Onychomycosis & Human Pathogen & 194339140 \\
\hline F. oxysporum & Onychomycosis & Human Pathogen & 194339144 \\
\hline F. oxysporum & Onychomycosis & Human Pathogen & 194339145 \\
\hline F. solani & Ocular sources & Human Pathogen & 198401847 \\
\hline F. solani & Ocular sources & Human Pathogen & 198401848 \\
\hline F. solani & Ocular sources & Human Pathogen & 198401867 \\
\hline F. solani & Ocular sources & Human Pathogen & 198401868 \\
\hline F. solani & Corneal Infections & Human Pathogen & 164654196 \\
\hline F. solani & Corneal Infections & Human Pathogen & 164654197 \\
\hline
\end{tabular}

- No data available, * Own isolates used in the present study.

of ITS sequence phylogeny did not yield evidence on variation of Fusarium species occupying different lifestyles, ITS2 consensus secondary structures were investigated for observation of discrepancy because structures are more conserved than sequences.

\section{ITS2 secondary structure prediction}

Consensus secondary structures of four different forms of Fusarium i.e. endophytes, saprobes, phytopathogens and human patho- gens were generated and comparison of structures among the diverse forms were studied. Endophytic forms showed close structural similarities with pathogenic forms but structural features of saprobic forms were totally different. The structural similarities of endophytic and pathogenic forms were observed in their longest helices which are arrowed in Figure 2. However, variations amongst them were seen in their respective junctions, hairpin loops, terminal and internal loops. The ITS2 
Plant Pathology \& Quarantine

consensus secondary structure analysis has pro-

vided information to conclude that endophytic

- Saprobe

- Phytopathogen

- Endophyte

A Human Pathogen

- F.oxysporum 183673382 Phytopathogen

O F.oxysporum 162285917 Saprobe

- F.oxysporum 68508669 Phytopathogen

- F.oxysporum 194339139 Human Pathogen

- F.oxysporum 260101097 Phytopathogen

- F.oxysporum 68508671 Phytopathogen

F.oxysporum 157922341 Phytopathogen

- F.oxysporum 205319826 Endophyte

A F.oxysporum 194339145 Human Pathogen

O F.oxysporum 148733622 Saprobe

O F.oxysporum 304651429 Saprobe

- F.oxysporum 302311065 Phytopathogen

A. Foxysporum 194339144 Human Pathogen

62 O F.oxysporum 148733625 Saprobe

- F.oxysporum 146217051 Endophyte

- F.oxysporum 82622243 Phytopathogen

O F.oxysporum 11692812 Saprobe

- Foxysporum 205319842 Saprobe

O F.oxysporum 304651428 Saprobe

O F.oxysporum 297185611 Saprobe

O F.oxysporum 204463578 Saprobe

- F.oxysporum 161876806 Phytopathogen

O F.oxysporum 60593117 Saprobe

O Foxysporum 289718953 Saprobe

- F.oxysporum 289119520 Saprobe

F.oxysporum 194580114 Phytopathogen

- F.oxysporum 116710911 Phytopathogen

- F.oxysporum 68267976 Phytopathogen

Foxysporum 194339140 Human Fathogen

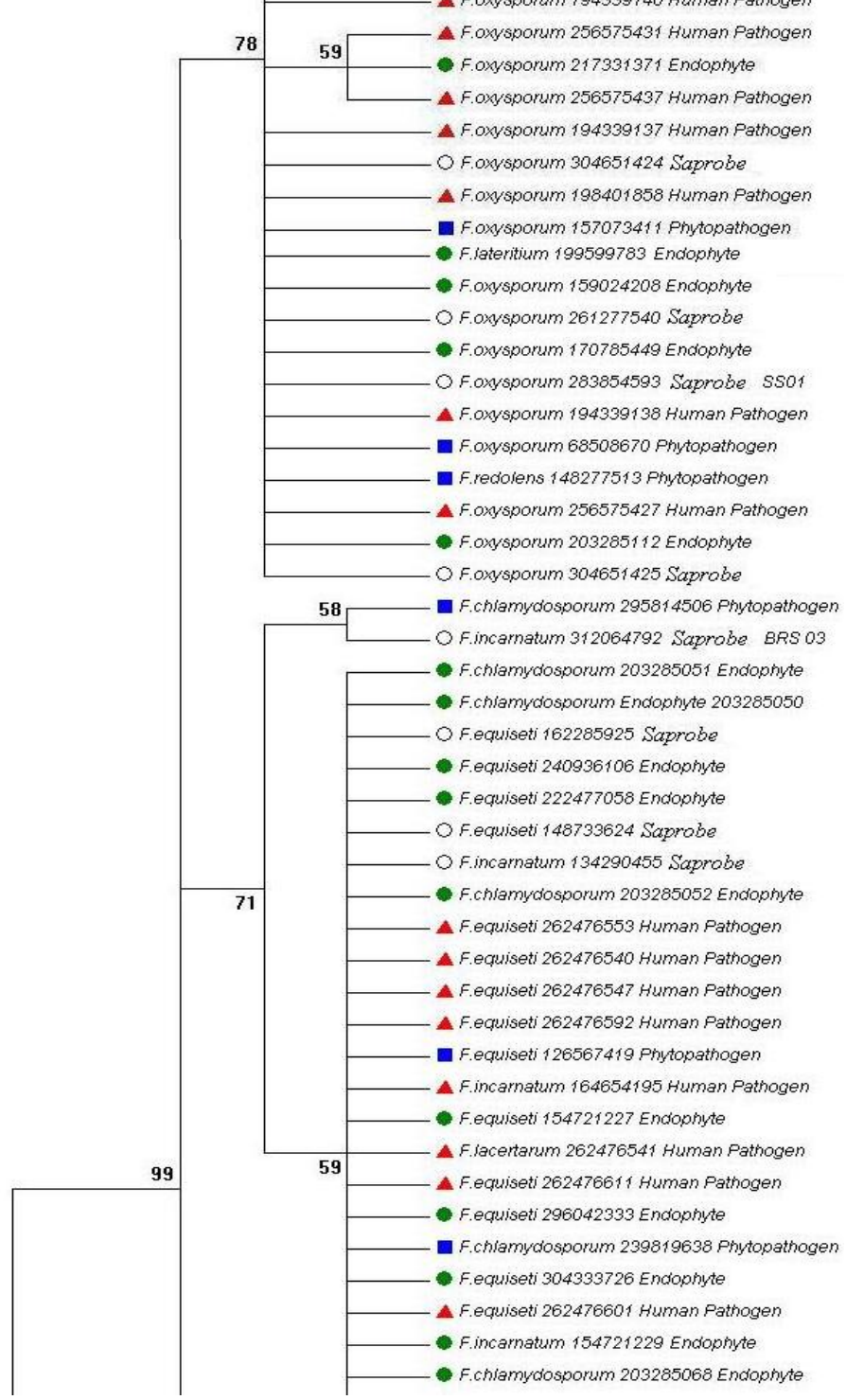




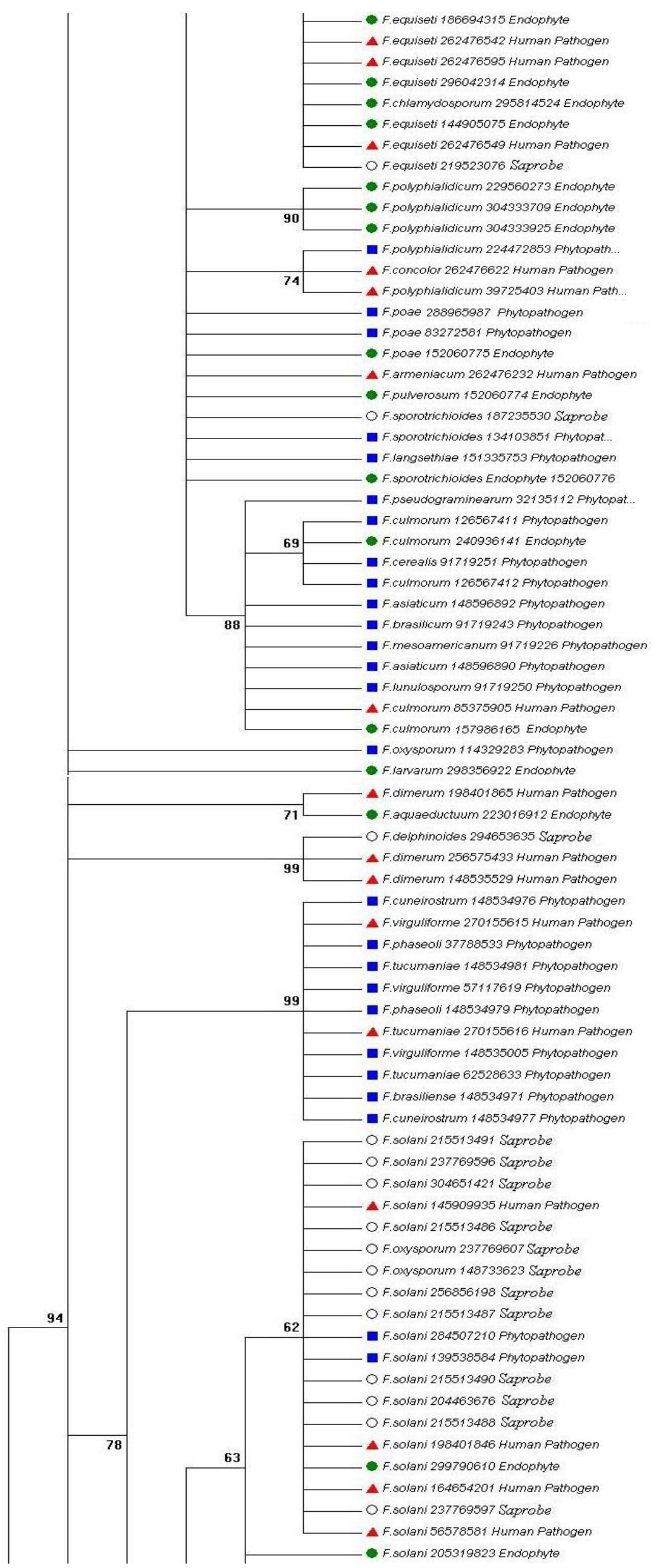


Plant Pathology \& Quarantine

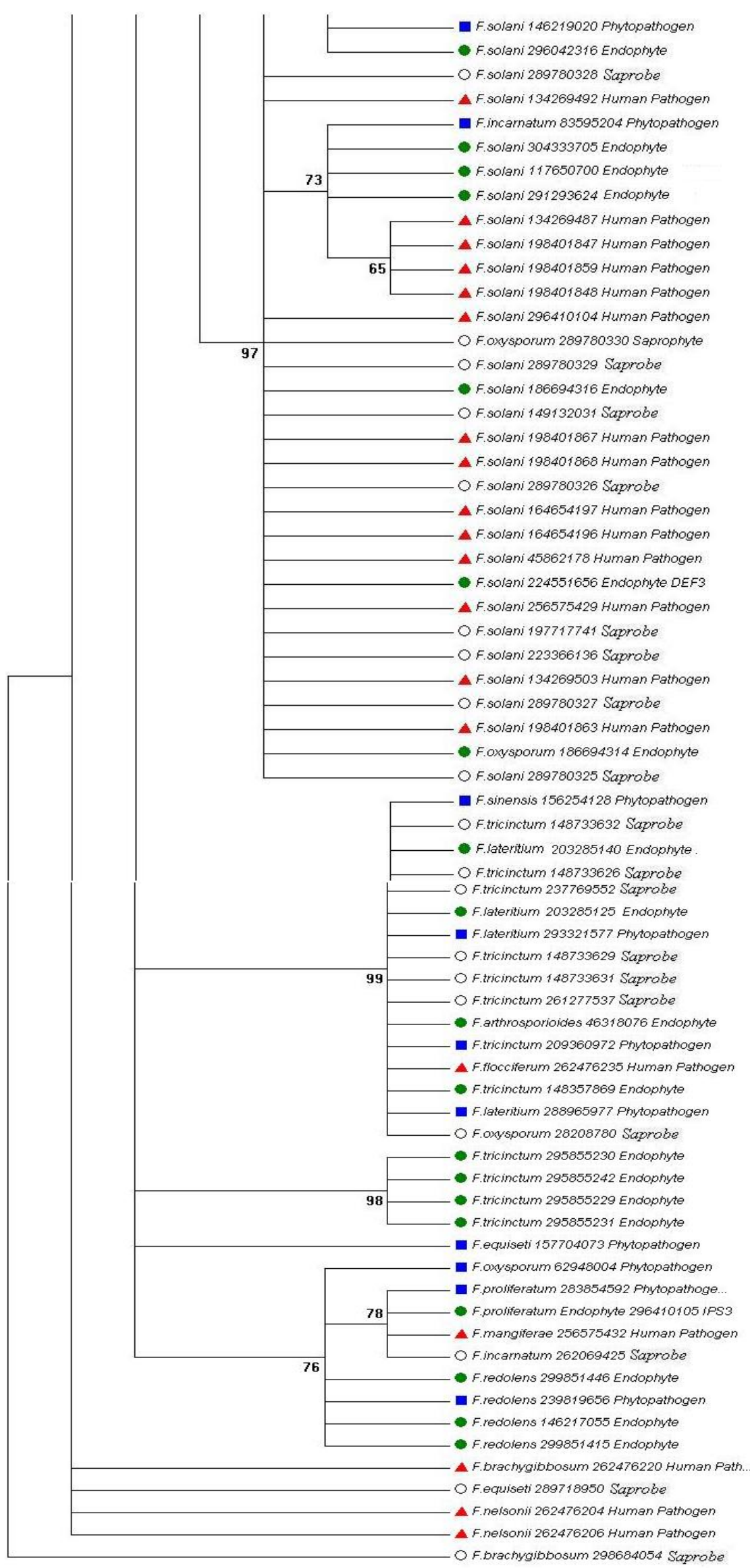


Fig. 1 - Phylogenetic tree showing evolutionary relationships of 212 Fusarium spp. of different lifestyles. The evolutionary history was inferred using the Maximum Parsimony method. The numbers indicate GenBank indices.

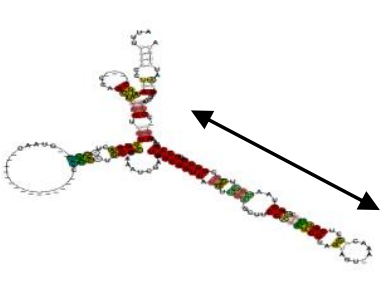

(a)

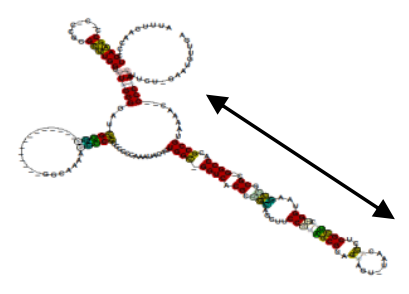

(c)

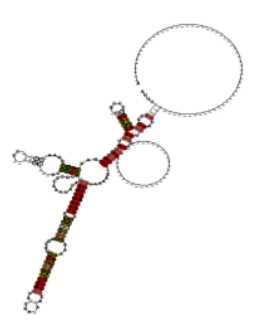

(b)

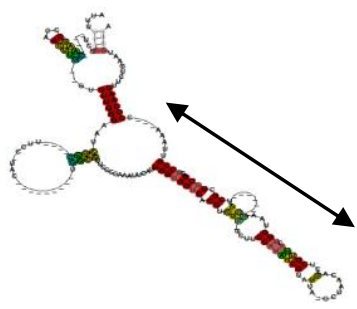

(d)

Fig. 2 - Predicted global consensus ITS2 RNA secondary structures of (a) endophytes, (b) saprobes, (c) Human Pathogens and (d) Phytopathogens. Compatible base pairs are colored, where the hue shows the number of different types C-G, G-C, A-U, U-A, G-U or U-G of compatible base pairs in the corresponding columns. In this way the hue shows sequence conservation of the base pair. The saturation decreases with the number of incompatible base pairs. Thus, it indicates the structural conservation of the base pair. The similar regions are arrow marked.

forms of Fusarium have close affinity with pathogenic forms while sequence based phylogeny did not show this result. Such findings agree with the assumption that endophytes are latent pathogens and close relationships exist between them (Sieber et al. 2007). There are numerous examples that endophytes become pathogens, however to our knowledge there is no molecular evidences to support their affinity.

\section{Acknowledgements}

The authors are grateful to Department of Biotechnology, Government of India for providing Bioinformatics Infrastructure Facility (BIF) and to the Coordinator BIF centre, North Orissa University, India for necessary support.

\section{References}

Ahvenniemi P, Wolf M, Lehtonen MJ, Wilson P, German-Kinnari M, Valkonen JP. 2009 - Evolutionary diversification indicated by compensatory base changes in
ITS2 secondary structures in a complex fungal species, Rhizoctonia solani. Journal of Molecular Evolution 69, 150-163.

Amata RL, Burgess LW, Summerell BA, Bullock S, Liew ECY, Smith-White JL. 2010 - An emended description of Fusarium brevicatenulatum and $F$. pseudoanthophilum based on isolates recovered from millet in Kenya. Fungal Diversity 43, 11-25.

Anderson IC, Parkin PI. 2007 - Detection of active soil fungi by RT-PCR amplification of precursor rRNA molecules. Journal of Microbiological Methods 68, 248253.

Albrectsen BR, Bjorken L, Varad A, Hagner A, Wedin M, Karlsson J, Jansson S. 2010 Endophytic fungi in European aspen $(\mathrm{Po}-$ pulus tremula) leaves-diversity, detection, and a suggested correlation with herbivory resistance. Fungal Diversity $41,17-28$. 
Bruns TD, White TJ, Taylor JW. 1991 - Fungal molecular systematics. Annual Review of Ecology and Systematics 22, 525-564.

Cai L, Jeewon R, Hyde KD. 2005 - Phylogenetic evaluation and taxonomic revision of Schizothecium based on ribosomal DNA and protein coding genes. Fungal Diversity $19,1-21$.

Cai L, Hyde KD, Taylor PWJ, Weir B, Waller J, Abang MM, Zhang JZ, Yang YL, Phoulivong S, Liu ZY, Prihastuti H, Shivas RG, McKenzie EHC, Johnston PR. 2009 - A polyphasic approach for studying Colletotrichum. Fungal Diversity 39, 183-204.

Coleman AW. 2003 - ITS2 is a double-edged tool for eukaryote evolutionary comparesons. Trends in Genetics 19, 370-375.

Coleman AW. 2007 - Pan-eukaryote ITS2 homologies revealed by RNA secondary structure. Nucleic Acids Research 35, 3322-3329.

Deng BV, Liu KH, Chen WQ, Ding XW, Xie XC. 2009 - Fusarium solani, Tax-3, a new endophytic taxol-producing fungus from Taxus chinensis. World Journal of Microbiology and Biotechnology 25, 139-143.

Freeman S, Rodrigues RJ. 1993 - Genetic conversion of a fungal plant pathogen to a nonpathogenic, endophytic mutualist. Science 260, 75-78.

Guo LD, Hyde KD, Liew ECY. 2001 - Detection and Taxonomic Placement of Endophytic Fungi within Frond Tissues of Livistona chinensis Based on rDNA Sequences. Molecular Phylogenetics and Evolution 20, 1-13.

Hausner G, Wang X. 2005 - Unusual compact rDNA gene arrangements within some members of the Ascomycota: evidence for molecular co-evolution between ITS1 and ITS2. Genome 48, 648-660.

Hyde KD, Soytong K. 2008 - The fungal endophyte dilemma. Fungal Diversity 33, 163-173.

Hyde KD, Bussaban B, Paulus B, Crous PW, Lee S, Mckenzie EHC, Photita W, Lumyong S. 2007 - Diversity of saprobic microfungi. Biodiversity and Conservation $16,7-35$.
Keller A, Schleicher T, Forster F, Ruderisch B, Dandekar T, Müller T, Wolf M. 2008 ITS2 data corroborate a monophyletic chlorophycean DO-group (Sphaeropleales). BMC Evolutionary Biology 8, 218.

Keller A, Schleicher T, Schultz J, Müller T, Dandekar T. 2009 - 5.8S-28S r RNA interaction and HMM-based ITS2 annotation. Gene 430, 50-57.

Kruger D, Gargas A. 2008 - Secondary structure of ITS2 rRNA provides taxonomic characters for systematic studies d a case in Lycoperdaceae (Basidiomycota). Mycological Research 112, 316-330.

Landis FC, Gargas A. 2007 - Using ITS2 secondary structure to create species- specific oligonucleotide probes for fungi. Mycologia 99, 681-692.

Miao M, Warrenb A, Songa W, Wangc S, Shanga H, Chena Z. 2008 - Analysis of the Internal Transcribed Spacer 2 (ITS2) Region of Scuticociliates and Related Taxa (Ciliophora, Oligohymenophorea) to Infer their Evolution and Phylogeny. Protist 159, 519-533.

Muller T, Philippi N, Dandekar T, Schultz J, Wolf M. 2007 - Distinguishing species. RNA 13, 1469-1472.

Nelson PE, Toussoun TA, Marasas WFO. 1983 - Fusarium species: An Illustrated Manual for Identification. Pennsylvania State University Press, University Park.

Nilsson RH, Ryberg M, Kristiansson E, Abarenkov K, Larsson KH, Koljalg U. 2006 - Taxonomic reliability of DNA sequences in public sequence databases: a fungal perspective. PLoS One 1, 59.

Photita W, Taylor PWJ, Ford R, Hyde KD, Lumyong S. 2005 - Morphological and molecular characterization of Colletotrichum species from herbaceous plants in Thailand. Fungal Diversity 18, 117-133.

Pinto PM, Resende MA, Koga-Ito CY, Ferreira JA, Tendler M. 2004 - rDNA- RFLP identification of Candida species in immunocompromised and seriously diseased patients. Canadian Journal of Microbiology 7, 514-520.

Promputtha I, Lumyong S, Dhanasekaran V, McKenzie EHC, Hyde KD, Jeewon R. 2007 - A phylogenetic evaluation of whether endophytes become saprotrophs 
at host senescence. Microbial Ecology 53, 579-590.

Promputtha I, Hyde KD, McKenzie EHC, Peberdy JF, Lumyong S. 2010 - Can leaf degrading enzymes provide evidence that endophytic fungi becoming saprobes? Fungal Diversity 41, 89-99.

Purahong W, Hyde KD. 2011 - Effects of fungal endophytes on grass and non-grass litter decomposition rates. Fungal Diversity $47,1-7$.

Redman RS, Ranson JC, Rodriguez RJ. 1999 Conversion of the pathogenic fungus Colletotrichum magna to a nonpathogenic, endophytic mutualist by gene disruption. Molecular Plant Microbe Interactions 11, 969-975.

Ruhl MW, Wolf M, Jenkins TM. 2010 - Compensatory base changes illuminate morphologically difficult taxonomy. Molecular Phylogenetics and Evolution 54, 664669.

Saikkonen K, Saari S, Helander M. 2010 - Defensive mutualism between plants and endophytic fungi? Fungal Diversity 41, 101-113.

Schroers HJ, O'Donnell K, Lamprecht SC, Kammeyer PL, Johnson S, Sutton DA, Rinaldi MG, Geiser DM, Summerbell RC. 2009 - Taxonomy and phylogeny of the Fusarium dimerum species group. Mycologia 101, 44-70.

Schulz B, Boyle C. 2005 - The endophytic continuum. Mycological Research 109, 661-686.

Schultz J, Wolf M. 2009 - ITS2 sequencestructure analysis in phylogenetics: A how to manual for molecular systematics. Molecular Phylogenetics and Evolution 52, 520-523.

Sieber T. 2007 - Endophytic fungi in forest trees: are they mutualists? Fungal Biology Reviews 21, 75-89.

Smith C, Heyne S, Richter AS, Will S, Back ofen R. 2010 - Freiburg RNA Tools: a web server integrating IntaRNA, ExpaRNA and LocaRNA. Nucleic Acids Research 1,38 .

Strobel, GA. 2002 - Microbial gifts from rain forests. Canadian Journal of Plant Pathology 24, 14-20.
Strobel GA, Daisy B. 2003 - Bioprospecting for Microbial Endophytes and Their Natural Products. Microbiology and Molecular Biology Reviews 67, 491502.

Summerell BA, Laurence MH, Liew ECY, Leslie JF. 2010 - Biogeography and phylogeography of Fusarium: a review. Fungal Diversity 44, 3-13.

Summerell BA, Leslie JF, Liew ECY, Laurence $\mathrm{MH}$, Bullock $\mathrm{S}$, Petrovic $\mathrm{T}$, Bentley AR, Howard CG, Peterson SA, Walsh JL, Burgess LW. 2011 - Fusarium species associated with plants in Australia. Fungal Diversity 46, 1-27.

Tamura K, Dudley J, Nei M, Kumar S. 2007 MEGA4: Molecular Evolutionary Genetics Analysis (MEGA) software version 4.0. Molecular Biology and Evolution 24, 1596-1599.

Tanaka A, Christensen MJ, Takemoto D, Park P, Scotta B. 2006 - Reactive oxygen species play a role in regulating a fungusperennial ryegrass mutualistic interacttion. Plant Cell 18, 1052-1066.

Tejesvi MV, Tamhankar SA, Kini KR, Rao VS, Prakash HS. 2009 - Phylogenetic analysis of endophytic Pestalotiopsis species from ethnopharmaceutically important medicinal trees. Fungal Diversity 38, 167-183.

Xu L, Zhou L, Zhao J, Li J, Li X, Wang J. 2008 - Fungal endophytes from Dioscorea zingiberensis rhizomes and their antibacterial activity. Letters in Applied Microbiology 46, 68-72.

Young I, Coleman AW. 2004 - The advantages of the ITS2 region of the nuclear rDNA Cistron for analysis of phylogenetic relationships of insects: a Drosophila example. Molecular Phylogenetics and Evolution 30, 236-242.

Wang Y, Guo, LD, Hyde, KD. 2005 - Taxonomic placement of sterile morphotypes of endophytic fungi from Pinus tabulaeformis (Pinaceae) in northeast China based on rDNA sequences. Fungal Diversity 20, 235-260.

Wei JG, Xu T, Guo LD, Liu AR, Zhang Y, Pan XH. 2007 - Endophytic Pestalotiopsis species associated with plants of Podocarpaceae, Theaceae and Taxaceae in 
southern China. Fungal Diversity 24, 5574.

White TJ, Bruns T, Lee S, Taylor JW. 1990 Amplification and direct sequencing of fungal ribosomal RNA genes for phylo- genetics. In: PCR Protocols, a guide to methods and applications (eds MA Innis, DH Gelfand, JJ Sninsky, TJ White). New York, Academic Press, 315-322. 